\title{
INDOOR MAPPING OF A COMPLEX CULTURAL HERITAGE SCENE USING TLS AND HMLS LASER SCANNING
}

\author{
V. E. Oniga ${ }^{1,2 *}$, A. I. Breaban ${ }^{1}$, E. I. Alexe ${ }^{1}$, C. Văsii ${ }^{3}$ \\ ${ }^{1}$ Department of Terrestrial Measurements and Cadastre, Faculty of Hydrotechnical Engineering, Geodesy and Environmental \\ Engineering, „Gheorghe Asachi” Technical University of Iasi, Professor Dimitrie Mangeron Boulevard 67, 700050, Iasi, Romania - \\ ersilia.oniga@tuiasi.ro,ioana.breaban@tuiasi.ro, eliza-iulia.alexe@student.tuiasi.ro \\ ${ }^{2}$ Romanian Society of Photogrammetry and Remote Sensing, Lacul Tei Blvd. 124, 020396, Bucharest, Romania - chair.srft@srft.ro \\ ${ }^{3}$ S.C. Helmert S.R.L, I.S. Sturza Boulevard 27, 600269, Bacau, Romania - cosmin.vasii@helmert.ro
}

\section{Commission II}

KEY WORDS: Terrestrial laser scanner, Point cloud, Registration, Hand-held Mobile laser scanner, Accuracy

\begin{abstract}
:
Indoor mapping and modelling is an important research subject with application in a wide range of domains including interior design, real estate, cultural heritage conservation and restoration. There are multiple sensors applicable for 3D indoor modelling, but the laser scanning technique is frequently used because of the acquisition time, detailed information and accuracy. In this paper, the efficiency of the Maptek I-Site 8820 terrestrial scanner, which is a long-range laser scanner and the accuracy of a HMLS point cloud acquired with a mobile scanner, namely GeoSlam Zeb Horizon were tested for indoor mapping. Aula Magna "Carmen Silva" of the „Gheorghe Asachi” Technical University of Iasi is studied in the current paper since the auditorium interior creates a distinct environment that combines complex geometric structures with architectural lighting and for preserving its great cultural value, the monument has a national historical significance. The registration process of the TLS point clouds was done using two methods: a semi-automatic one with artificial targets and a completely automatic one, based on Iterative Closest Point (ICP) algorithm. The resulted TLS point cloud was analysed in relation to the HMLS point cloud by computing the M3C2 (Multiscale Model to Model Cloud Comparison), obtaining a standard deviation of $2.1 \mathrm{~cm}$ and by investigating the Hausdorff distances from which resulted a standard deviation $(\sigma)$ of $1.6 \mathrm{~cm}$. Cross-sections have been extracted from the HMLS and TLS point clouds and after comparing the sections, $80 \%$ of the sigma values are less or equal to $1 \mathrm{~cm}$. The results show high potential of using HMLS and also a long-range laser scanner for 3D modelling of complex scenes, the occlusion effect in the case of TLS being only 5\% of the scanned area.
\end{abstract}

\section{INTRODUCTION}

Laser scanning technique is often used for mapping complex scenes, both indoor and outdoor, the resulted point cloud being transformed into a three-dimensional (3D) model that represents with high fidelity the subject of interest, making the analysis of geometrical details easier which is, with traditional techniques, difficult to achieve. For restoration or conservation projects of cultural heritage sites, the $3 \mathrm{D}$ model is essential and a high accuracy must be assured.

A 3D model of an indoor complex scene obtained from a static terrestrial laser scanner (TLS) requires multiple scans to avoid occlusions and to obtain a consistent indoor scene model. Nevertheless, some indoor parts are missing from the final point cloud and for a completed model, additional data have to be collected and integrated. When using a mobile hand-held laser scanner (HMLS), the data acquisition time is relatively short around a few minutes and is mainly influenced by the scene size. In contrast, the resulted point cloud has an accuracy usually inferior to that of the TLS point cloud. Other drawbacks of using hand-held mobile laser scanner were stated by Velas et al. (2019): the sensor has to be in constant movement and is sensitive to motions in the scene.

In several studies the accuracy of the HMLS point cloud is evaluated in terms of distances between each point and the best fitting geometrical shapes. In the experiment conducted by Maboudi et al. (2017), the standard deviation of the distances between points and the best fitting planes was $11 \mathrm{~mm}$. Considering two perfectly planar surfaces of around $30 \mathrm{~m}^{2}$, scanned with a Zeb-Revo handheld mobile laser scanner, the point-to-plane distances had a maximum of $\pm 25.5 \mathrm{~mm}$ and 32.2 $\mathrm{mm}$, respectively (Dewez et al., 2016).

Over the last years, many comparative studies between TLS and HMLS point clouds have been conducted, for forest inventory applications (Gollob et al., 2020; Shao et al., 2020; Cabo et al., 2018; Bauwens et al., 2016; Ryding et al., 2015) and indoor mapping (Russhakim et al., 2019; Sirmacek et al., 2016). The comparison was made in terms of specific elements size, such as: tree height, diameter at breast height, segments lengths etc. not for the entire point clouds, concluding that the derived future elements from HMLS point clouds could not achieve the same quality of geometrical accuracy as TLS, but the differences are not notable.

The aim of this research is, firstly, to test the efficiency in 3D modelling of indoor scenes of a long-range laser scanner i.e., Maptek I-Site 8820, designed especially for mining applications and secondly, to evaluate the accuracy of a HMLS point cloud acquired with a mobile hand-held laser scanner, namely GeoSlam Zeb Horizon.

\footnotetext{
* Corresponding author: Valeria-Ersilia Oniga: ersilia.oniga@tuiasi.ro
} 


\section{MATERIAL AND METHOD}

\subsection{Study area}

The scene under study is a large auditorium called Aula Magna "Carmen Silva" located inside the University Palace of Copou in Iasi city and the displayed classical baroque architectural style increases the historical value of the monument, making from the remarkable Aula Magna one of the most beautiful auditorium in the country. Its history is linked to that of the building where it is located. Twelve portraits were painted on the ceiling in 1958 and two portraits representing King Carol I and Queen Elizabeth (Carmen Sylva) were placed here in 2013. Individualizing through the spherical shape and glass ceiling, the high number of banks, statues placed on the walls and architectural elements, the "Carmen Silva" auditorium is considered a very complex scene.

\subsection{Data Acquisition Using Maptek I-Site 8820}

The Maptek I-Site 8820 laser scanner, suited for many indoor or outdoor application, especially mining environment (stockpile volumes, pit surveys), can be used either for static or mobile scanning, combining very high measurement accuracy with reliability and flexibility. The scanner is produced by Maptek company in Adelaide, south Australia. The scanning range is maximum $2000 \mathrm{~m}$, the maximum vertical field of view is $80^{\circ}$ and the horizontal field of view (FOV) is $360^{\circ}$. Having an integrated 70-megapixel panoramic digital camera, the colour texture of the scanned scene is automatically transferred to the resulted point cloud (Keawaram and Dumrongchai, 2017).

For the present study, a number of 7 scans have been acquired using the TLS laser scanner in approximately 3 hours (Figure 1 a), the number 4 being chosen to define the point cloud resolution, as can be seen in Figure $1 \mathrm{~b}$.

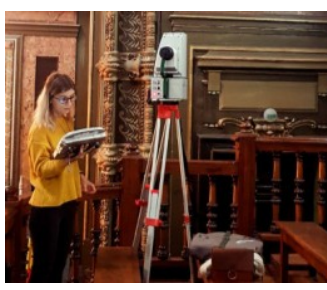

(a) (b)

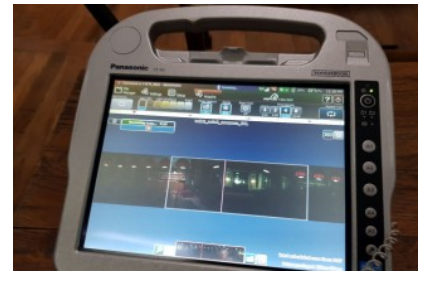

Figure 1. Data acquisition using (a) the Maptek I-Site 8820 terrestrial laser scanner; (b) controller tablet

The Maptek I-Site 8820 resolution is defined by four numbers i.e., $1,2,4,8$ and 16 which, according to the technical specifications the values for the azimuth and elevation resolution, the number of scan lines and the time to complete a $360^{\circ}$ scan are summarized in Table 1.

\begin{tabular}{|c|c|c|c|c|}
\hline $\begin{array}{c}\text { Point } \\
\text { density } \\
\text { setting }\end{array}$ & $\begin{array}{c}\text { Azimuth } \\
\text { resolution } \\
\text { (Degrees } \\
\text { per step) }\end{array}$ & $\begin{array}{c}\text { Elevation } \\
\text { resolution } \\
\text { (Degrees } \\
\text { per step) }\end{array}$ & $\begin{array}{c}\text { No. of } \\
\text { scan } \\
\text { ines } \\
\text { per } \\
360^{0}\end{array}$ & $\begin{array}{c}\text { Time to } \\
\text { complete } \\
360^{\circ} \\
\text { scan @ 4 40 } \\
\mathrm{kHz} \text { (min.) }\end{array}$ \\
\hline 1 & 0.2 & 0.1 & 1,800 & 3.4 \\
\hline 2 & 0.1 & 0.1 & 3,600 & 6.1 \\
\hline 4 & 0.05 & 0.05 & 7,200 & 22.3 \\
\hline 8 & 0.025 & 0.025 & 14,400 & 87.0 \\
\hline 16 & 0.0125 & 0.0125 & 28,800 & NA \\
\hline
\end{tabular}

Table 1. Point density settings for Maptek I-Site 8820 laser scanner
The data points with intensity attribute captured by the terrestrial laser scanner for the presidium area (Figure 2 a) can be viewed in Figure 2 b.

Being a relatively dark room, even if the lights have been open, the RGB code of the point clouds is not offering a very good understanding of the details. As such, a pre-processing step was done using the "Point Studio" software by adjusting the images colour code. The point cloud with the modified RGB colour code can be viewed in Figure $2 \mathrm{c}$ and in greyscale colour palette in Figure $2 \mathrm{~d}$.

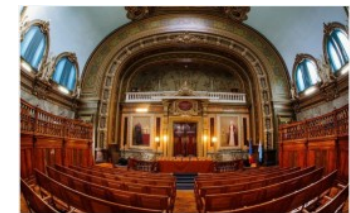

(a)

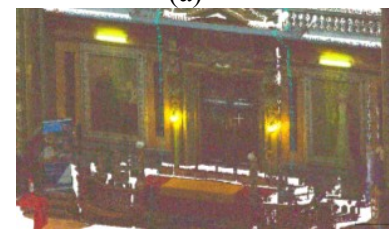

(c)

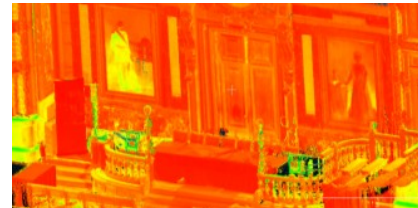

(b)

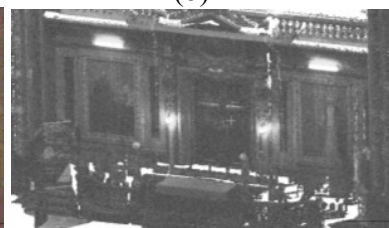

(d)
Figure 2. (a) Image representing the balcony of "Carmen Silva" auditorium and the presidium area; (b) TLS point cloud with intensity attribute; (c) TLS point cloud with RGB colour code;

(d) TLS point cloud in greyscale colour palette

Six spheres have been placed in the interior of the auditorium to ensure the visibility from each scan station, three of them being placed on tripods with the help of tribrachs (Figure 3 a) and three of them on the upper railing. The location of the laser scanner station points and of the six spheres, have been represented in Figure 3 b. Also, two floor lamps with the shape of spheres located in the presidium area that were used for the automatic registration process accuracy assessment, are represented in Figure $3 \mathrm{~b}$.

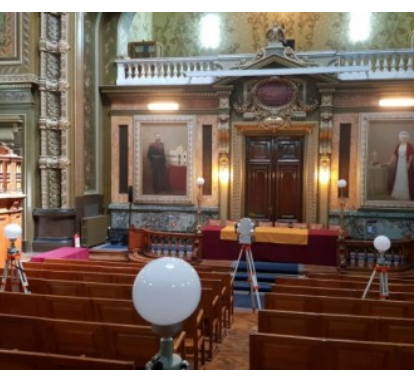

(a)

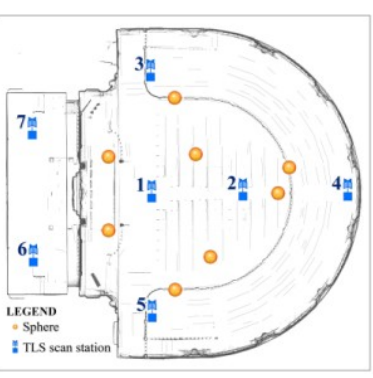

(b)
Figure 3. (a) Artificial spheres with $10 \mathrm{~cm}$ diameter used for TLS point clouds registration; (b) locations of TLS scan stations and spheres

\subsection{Data Acquisition Using GeoSlam Zeb Horizon}

The GeoSlam Zeb Horizon hand-held laser scanner, makes the three-dimensional measuring and the digital data capturing very easy, being able to collect and process the scanning data simultaneously simply by walking. Can be used also for indoor and outdoor mapping, scanning in an FOV of $360^{\circ} \times 270^{\circ}$ at a maximum range of $100 \mathrm{~m}$ and collecting approximately 300000 points $/ \mathrm{s}$ with a relative accuracy of $1-3 \mathrm{~cm}$, as can be found in the laser specifications (GeoSLAM Ltd., Nottingham, UK) (Figure 4). 
When using the HMLS technology, the "Carmen Silva" auditorium was scanned in 3 minutes and 6 seconds obtaining a number of approximately 48 millions points.

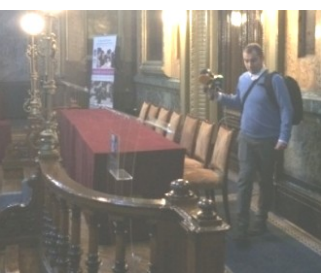

(a)

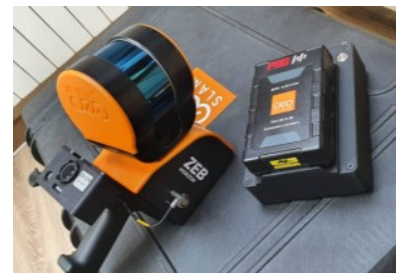

(b)
Figure 4. (a) Data acquisition; (b) the GeoSLAM Zeb-Horizon 3D mobile Scanner

\subsection{TLS point clouds registration}

In order to bring into the same coordinate system, the individual scans, two registration methods have been applied. The first one is an automatic method based on Iterative Closest Point (ICP) algorithm (Besl et al., 1992) and the second one is a semiautomatic method based on 3D conformal transformation (Oniga et al., 2016).

\subsubsection{Automated registration}

The first registration algorithm applied to the 7 individual scans, is fully automatic and was implemented into "Point Studio" software. The process was done performing the following steps:

- initial alignment of the first point cloud pair using the "Automated initial positioning" function, from "Position and Filter" menu;

- global registration of the initial aligned point clouds using the ICP algorithm;

- merging the registered point clouds;

- continue the operation by progressively adding new scans.

The point cloud considered as reference for the registration process was the first point cloud, number 1 , as it was acquired from the centre of the auditorium with $360^{\circ}$ horizontal FOV.

The accuracy of each registration algorithm was evaluated by using the Root Mean Square Error (RMSE) and the standard deviation as a result of comparing the overlapping areas. Both statistical measures are automatically calculated and listed by the "Point Studio" software.

We have to mention that only checking the values of these two statistical measures, the one can get a wrong idea about the accuracy of the registration process. In Figure 5 are coloured with two different colours the point clouds acquired from two different station on a common sphere. Even if the registration errors are small, respectively $1 \mathrm{~cm}$ for RMSE and $5 \mathrm{~mm}$ for the standard deviation, visually we can see that the point clouds are not fitting.

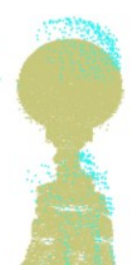

Figure 5. Point clouds measured on a sphere mounted on a tripod from two different positions
So, in order to evaluate the accuracy of the registration process done automatically, we will also compute the euclidian distance between the centres of the best fitting spheres found for each point cloud. In Table 2 are summarized the RMSE, the standard deviation $(\sigma)$ and the RMSE of the differences between the spheres centres.

\begin{tabular}{|c|c|c|c|}
\hline \multirow[b]{2}{*}{ Pair of point clouds } & \multicolumn{2}{|c|}{ Errors } & \multirow{2}{*}{$\begin{array}{c}\text { RMSE } \\
\text { of } \\
\text { euclidian } \\
\text { distances } \\
\text { [mm] }\end{array}$} \\
\hline & $\begin{array}{c}\text { RMSE } \\
\text { [mm] }\end{array}$ & $\underset{[\mathbf{m m}]}{\sigma}$ & \\
\hline $1+2$ & 5 & 4 & 10 \\
\hline$(1+2)+3$ & 7 & 4 & 8 \\
\hline$(1+2+3)+4$ & 7 & 4 & 8 \\
\hline$(1+2+3+4)+5$ & 7 & 4 & 12 \\
\hline$(1+2+3+4+5)+6$ & 13 & 5 & 28 \\
\hline$(1+2+3+4+5+6)+7$ & 10 & 5 & 25 \\
\hline$(1+2+3+4+5+6+7)+8$ & 10 & 5 & 23 \\
\hline
\end{tabular}

Table 2. The errors of the TLS point clouds registration process computed automatically by "Point Studio" software and the RMSE of the euclidian distances between the spheres geometrical centres

The spheres coordinates have been obtained after finding the best fitting spheres for the corresponding point cloud (Figure 6), using the least square method implemented into "CloudCompare" software.

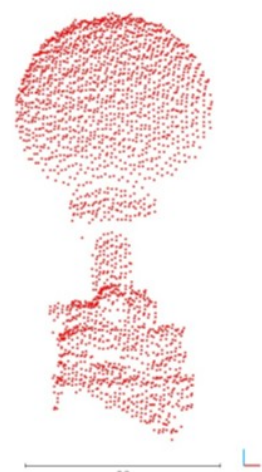

(a)

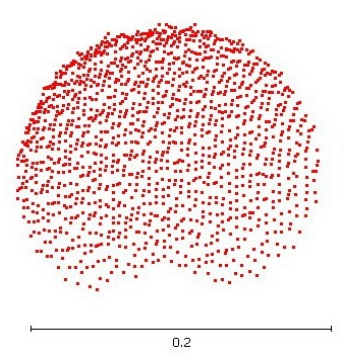

(b)

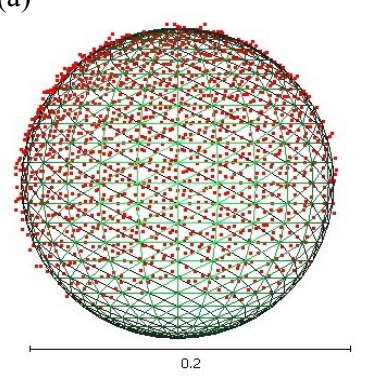

(c)
Figure 6. (a) TLS point cloud corresponding to the artificial sphere mounted on a tribrach; (b) segmented TLS points; (c) best fitting sphere

The point cloud obtained after the registration process can be visualized in grey scale colour palette (Figure 7 a) and with RGB colour code (Figure 7 b). 


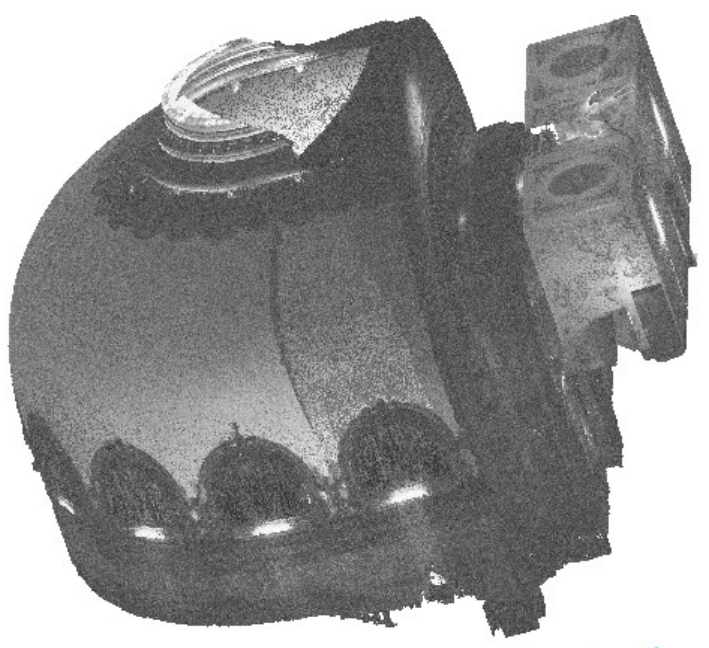

(a)

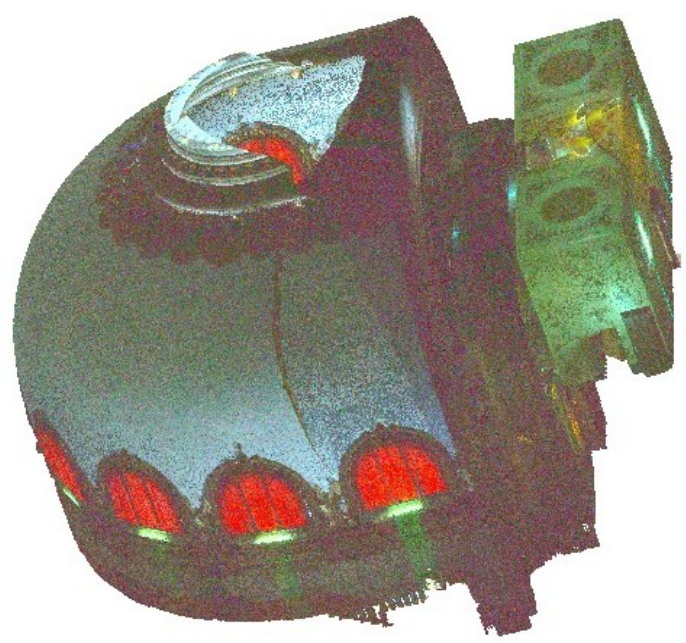

(b)

Figure 7. The resulted point cloud represented (a) in grey scale colour palette; (b) RGB colour

The registered point cloud was in a local coordinate system with the origin at the first scanning position placed in the auditorium centre, the scanning process being performed in $360^{\circ}$ horizontal FOV.

\subsubsection{Semi-automated registration using artificial targets}

The registration process for the TLS point clouds was done using the $3 \mathrm{D}$ conformal transformation implemented into "CloudCompare" software by choosing the "Registration Align (point pairs picking)" function and for each pair a RMSE less or equal to $10 \mathrm{~mm}$ was obtained (Table 3 ).

\begin{tabular}{|c|c|c|}
\hline \multirow{2}{*}{$\begin{array}{c}\text { Point clouds } \\
\text { pair }\end{array}$} & \multicolumn{2}{|c|}{ Errors } \\
\cline { 2 - 3 } & $\begin{array}{c}\text { RMSE } \\
{[\mathbf{m m}]}\end{array}$ & $\begin{array}{c}\boldsymbol{\sigma} \\
{[\mathbf{m m}]}\end{array}$ \\
\hline $1+2$ & 3 & 5 \\
\hline$(1+2)+3$ & 4 & 2 \\
\hline$(1+2+3)+4$ & 6 & 3 \\
\hline$(1+2+3+4)+5$ & 3 & 5 \\
\hline$(1+2+3+4+5)+6$ & 10 & 6 \\
\hline$(1+2+3+4+5+6)+7$ & 10 & 3 \\
\hline$(1+2+3+4+5+6+7)+8$ & 10 & 6 \\
\hline
\end{tabular}

Table 3. The errors of each individual registration process

\subsection{HMLS point clouds registration}

In the case of the GeoSlam Zeb Horizon hand-held laser scanner, the registration process was performed automatically with the Simultaneous Localization and Mapping (SLAM) algorithm, implemented into GeoSLAM Hub 6.3 software. The registered point cloud is given in a local coordinate system, with origin at the start/end position of the walking path that can be seen in Figure 8.

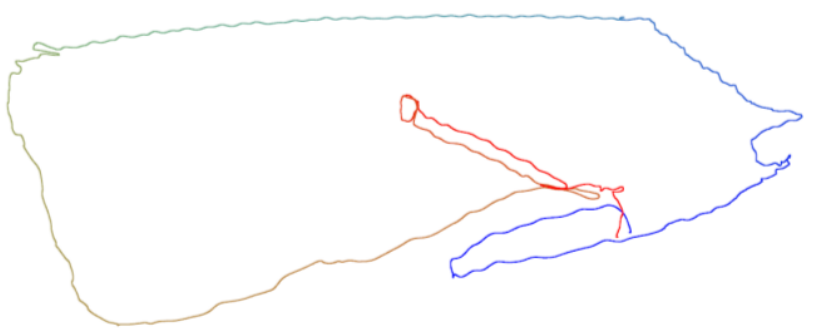

Figure 8. The path of Zeb Horizon sensor for auditorium scanning process

The HMLS point cloud, resulted after applying a noise filter using the GeoSLAM Hub 6.3 software is presented in Figure 9 in shade colour palette.

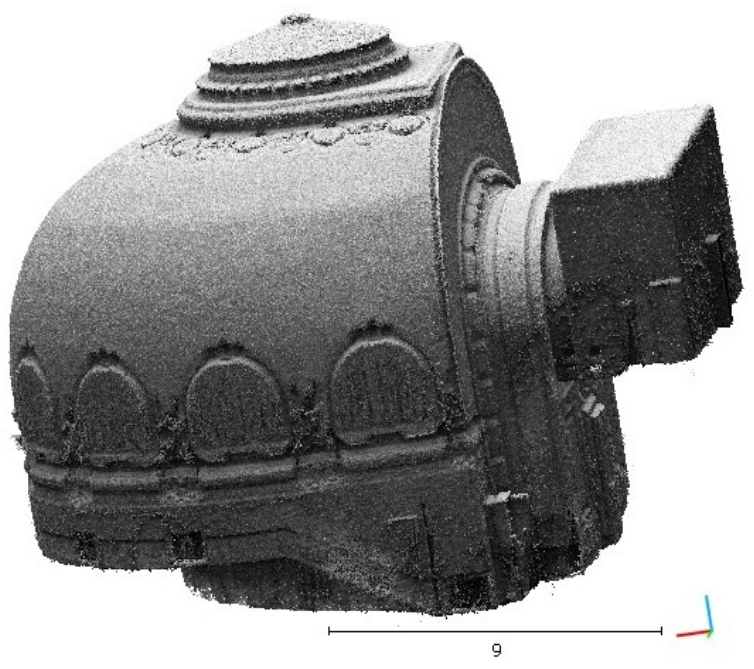

(a)

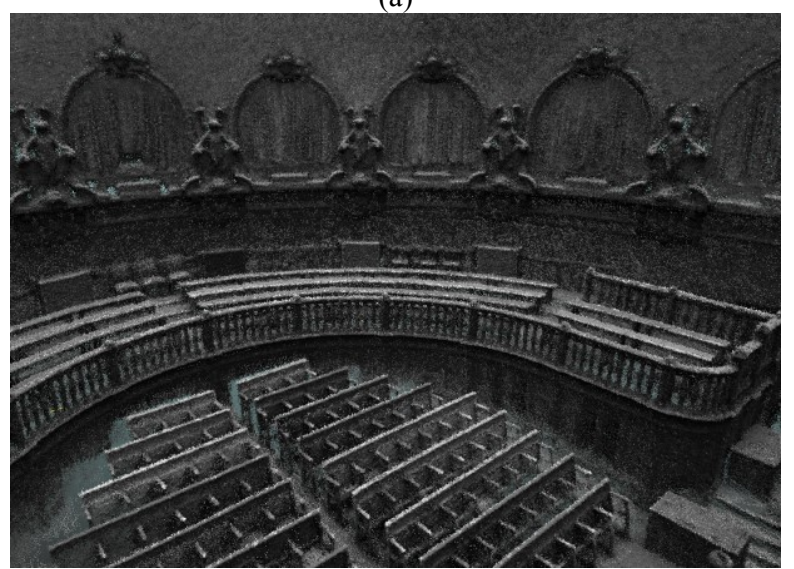

(b)

Figure 9. HMLS point cloud after noise reduction (a) a perspective view of the auditorium; (b) detail with benches 


\section{RESULTS AND DISCUSSIONS}

\subsection{TLS and HMLS point cloud comparison}

For the comparison, the TLS and the HMLS point clouds were brought into the same coordinate system. First, an initial alignment was performed by using the $3 \mathrm{D}$ conformal transformation and five common spheres, two of them representing the floor lamps with the shape of spheres located in the presidium area and three of them situated on the upper railing, the RMSE being $1.4 \mathrm{~cm}$. Then, the final registration was done using the ICP algorithm implemented into "PointStudio" software obtaining a RMSE of $9 \mathrm{~mm}$.

The HMLS point cloud was compared with the TLS point cloud by computing the $\mathrm{M} 3 \mathrm{C} 2$ (Multiscale Model to Model Cloud Comparison) distances implemented into "CloudCompare" software, by subsampling the point cloud at $0.1 \mathrm{~m}$, obtaining a standard deviation of $2.1 \mathrm{~cm}$ (Figure 10).

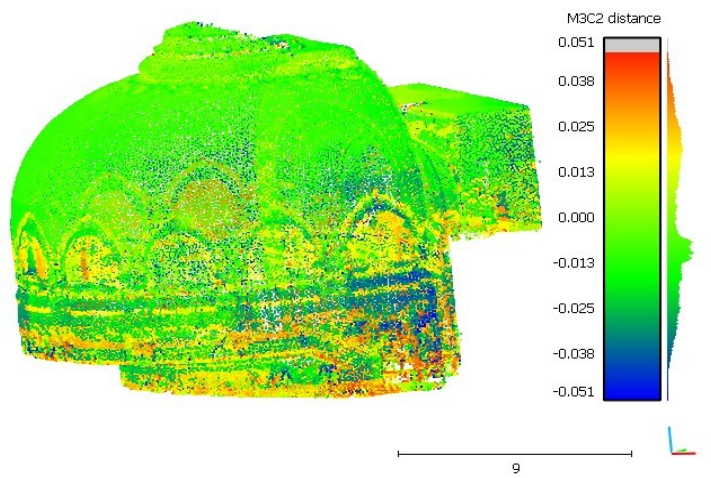

Figure 10. HMLS points coloured based on M3C2 distances between each point and the TLS point cloud

Because of computational memory, the TLS point cloud was resampled at a minimum distance of $5 \mathrm{~cm}$ and a mesh surface was created using the "Spherical Surface" function from "Point Studio" software. The resulted mesh surface was considered reference for the second comparison. So, using the "DistanceCloud/Mesh Dist" function implemented into "CloudCompare" software, the Hausdorff distances between each HMLS point and the corresponding TLS triangle surface were calculated. After removing the points that don't have a correspondent into the mesh surface based on the histogram of the calculated distances, in number of approximately 2 millions points, like stairs, benches, the middle part of the ceiling, the area behind the presidium table (Figure 11), the distances range was $-5.5 \mathrm{~cm}-+3.0 \mathrm{~cm}$ and the standard deviation of $1.6 \mathrm{~cm}$ calculated by fitting the Gauss distribution (Figure 12).

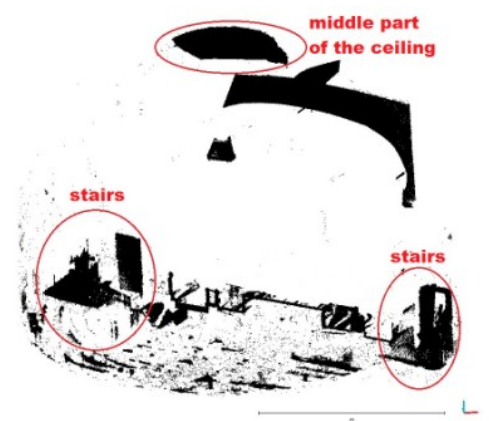

Figure 11. HMLS points representing the missing areas from the TLS point cloud

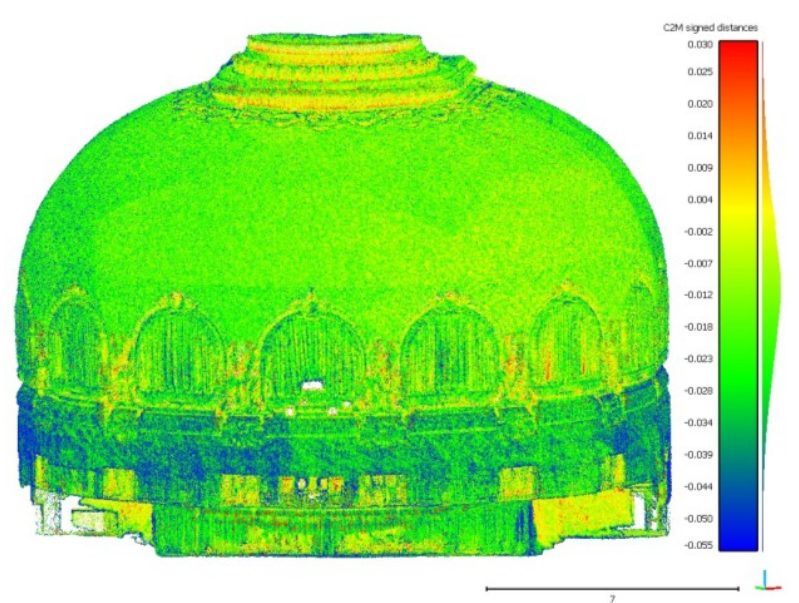

Figure 12. The Hausdorff distances calculated for the HMLS point cloud using as reference data the mesh created based on TLS point cloud

Also, the HMLS point cloud accuracy was tested by finding the best fitting geometrical shapes such as: sphere and plane (Figure 13). The standard deviation of the distances between HMLS points and the best fitting geometrical shapes is $1.5 \mathrm{~cm}$ and the maximum distance between a HMLS point and the best fitting geometrical shape is about $5 \mathrm{~cm}$.

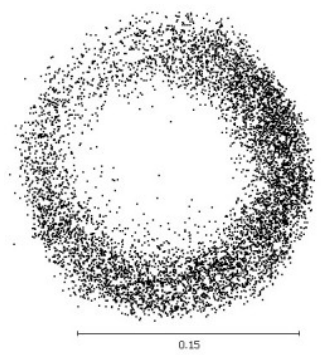

(a)

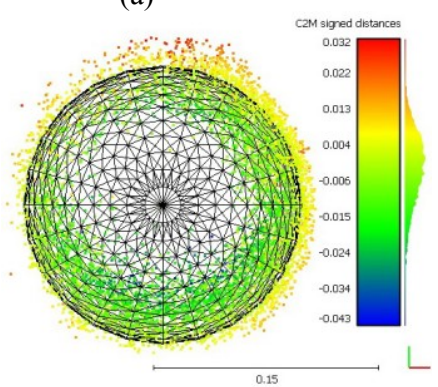

(c)

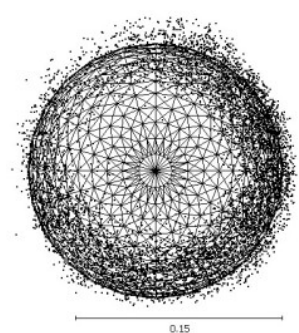

(b)

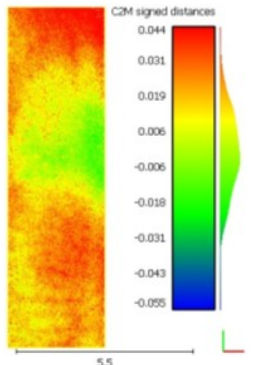

(d)
Figure 13. Finding the best fitting geometrical shapes (a) HMLS point cloud representing a floor lamp; (b) best fitting sphere; (c) HMLS points coloured based on perpendiculars between each point and best fitting sphere; (d) HMLS points coloured based on perpendiculars between each point and the best fitting plane

\subsection{Cross-sections comparison}

The GeoSlam Zeb Horizon point cloud is often used to extract profiles and sections of the scanned scene, so a comparison in terms of distances between two sections extracted for TLS and HMLS point cloud respectively was made.

Both point clouds have been rotated around the $\mathrm{Z}$ axis, in order to align the best fitting plane for the points measured on the 
back wall with the $\mathrm{Y}$ axis. Starting from this plane and parallel with it, have been defined section lines (Figure 14 a) at equal distances i.e., $0.5 \mathrm{~m}$, obtaining a total of 38 sections (Figure 14 b, c and d).

For the TLS point cloud, all the steps have been taken into "CloudCompare" software, using the "Cross section" and "Extract sections" functions from the "Segmentation" menu. The cross-sections defined as polylines have been exported as *.dxf for further comparison with the cross-sections extracted from the HMLS point cloud.

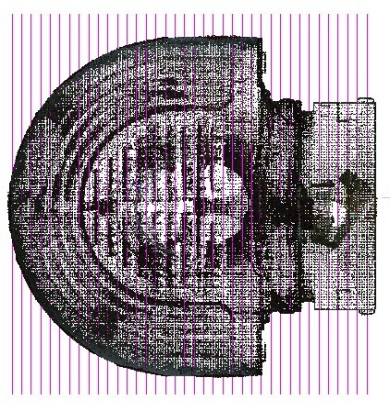

(a)

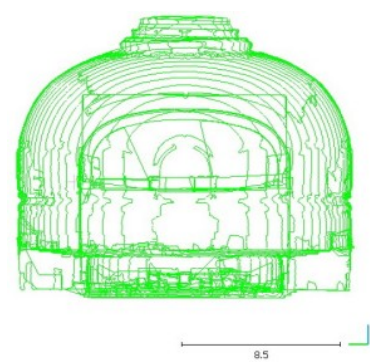

(c)

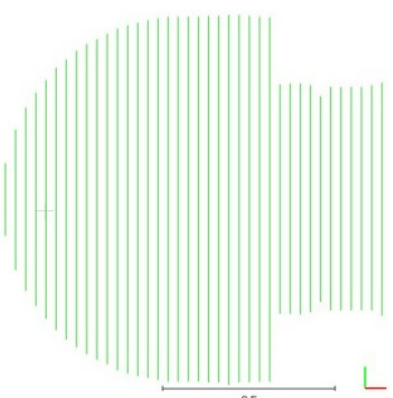

(b)

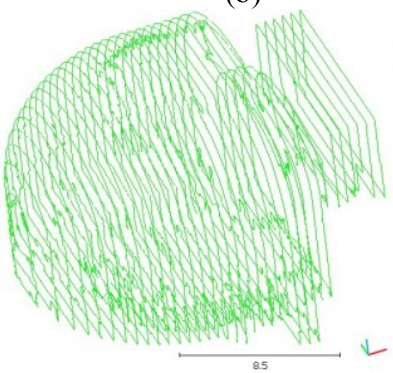

(d)
Figure 14. (a) Defining sections lines and extraction of crosssection for the TLS point cloud; (b) top view; (c) front view; (d) perspective view

For the HMLS point cloud, the above-described steps have been implemented in "Reconstructor 4.3" software (Figure 15).

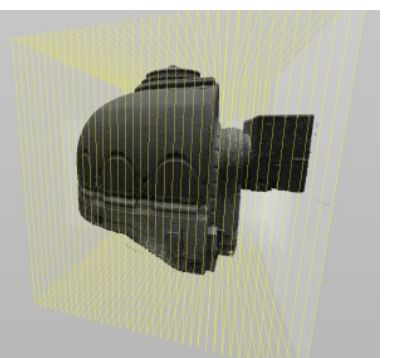

(a)

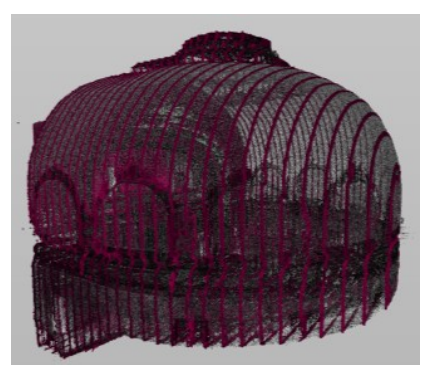

(b)
Figure 15. (a) Defining sections lines and extraction of crosssections for the HMLS point cloud at equal distances of $0.5 \mathrm{~m}$ in Reconstructor 4.3 software; (b) cross-sections polylines superimposed on the HMLS point cloud

Each polyline was divided in 20 approximately equal segments. The defined segments were used for measuring the distances between the TLS and HMLS cross-sections for which the standard deviation and the RMSE were computed. $80 \%$ of the $\sigma$ values are less or equally to $1 \mathrm{~cm}$ and $90 \%$ of the RMSE values are less or equally to $1.5 \mathrm{~cm}$ which is also the standard deviation obtained for the best fitting geometrical shapes.
For a deep analysis of the architectural elements, horizontal cross-sections can be derived based on a point cloud with a specified width using the GeoSLAM Draw software. Figure 16 represents a horizontal cross-section for the bottom half of the auditorium.

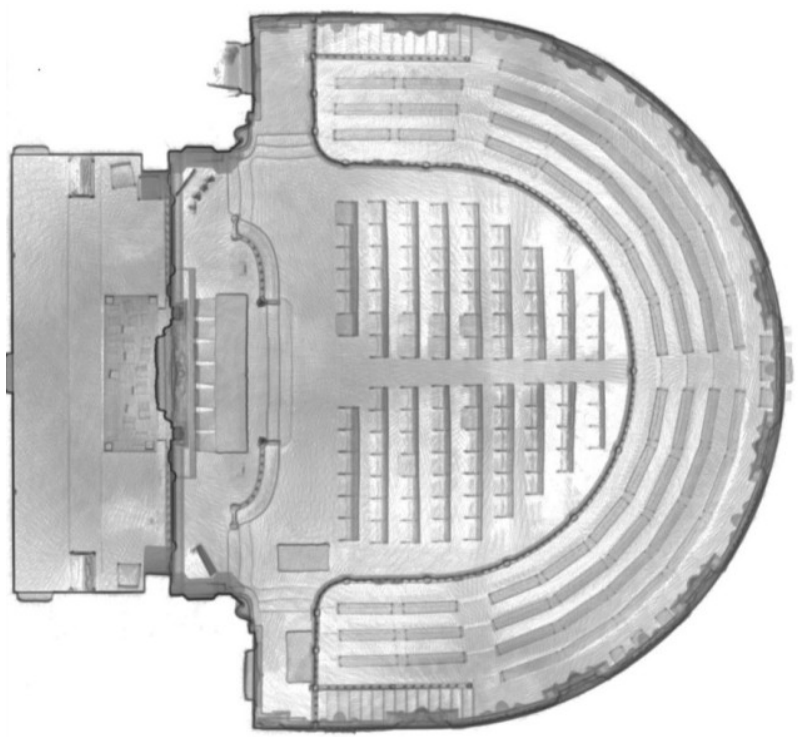

Figure 16. Horizontal cross-section of the lower part of the auditorium

\subsection{D modelling of the auditorium}

For the restoration process, the main purpose was to create the 3D model of the entire auditorium and the detailed 3D model of a statue and of all representative architectural elements. So, the first step in point cloud processing was the noise filtering which was done automatically using the "Noise filter" function of "CloudCompare" software.

As can be seen from Figure 11, some parts are missing from the TLS point cloud and in order to have a complete scene, an integration of TLS and HMLS point clouds was done. The missing parts represent approximately $5 \%$ of the scanned scene and are highlighted in red in the integrated point cloud (Figure 16).

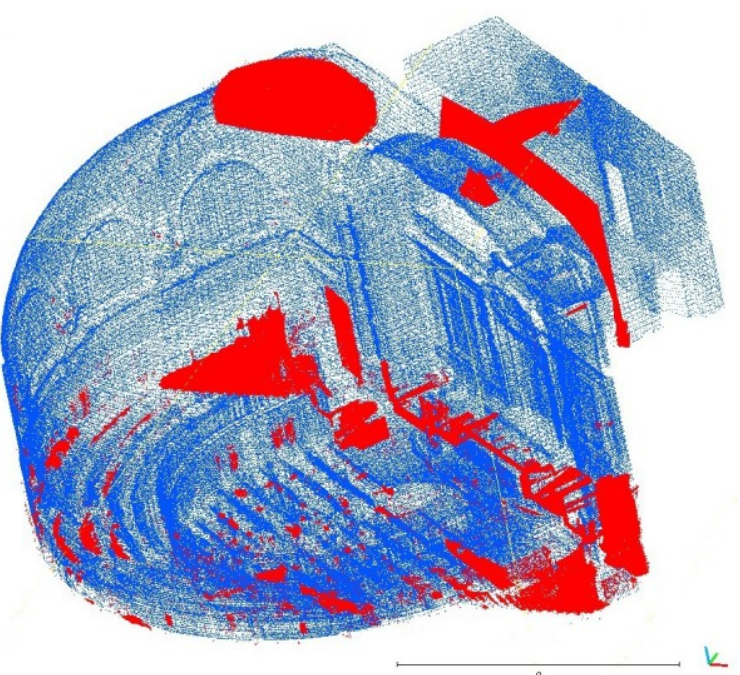

Figure 16. Integrated point cloud of the auditorium (TLS point cloud coloured in blue and HMLS point cloud coloured in red) 
The 3D model of the entire scene was created as a mesh surface, and the "MeshLab" software was used for mesh creation. After testing different methods, the Poisson method gave the best results, thus was adopted for the present study. A final step in mesh processing was to apply the Laplacian operator to smooth the entire result. Figure 17 represents the 3D model of one of the statues placed between the auditorium windows.

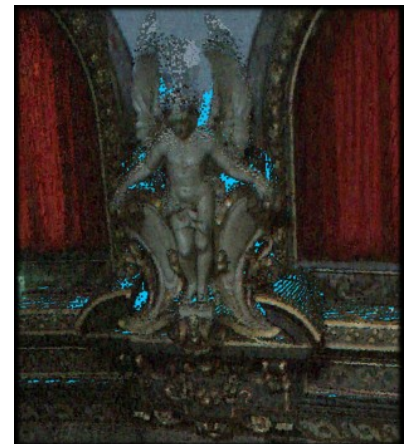

(a)

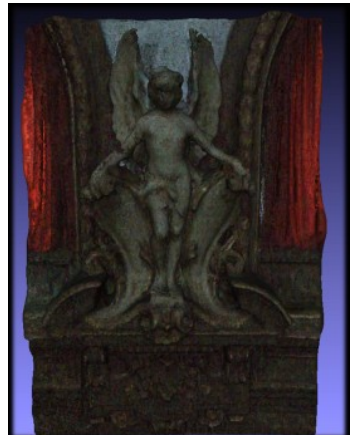

(b)
Figure 17. (a) Integrated point cloud detail of a statue; (b) mesh surface created with Poisson method and Laplacian Smoothing function

Some representative architectural elements of the auditorium modelled as a mesh surface, the wall from the back of the presidium area, the ceiling and the arch that frames the presidium area, can be seen in Figure 18.

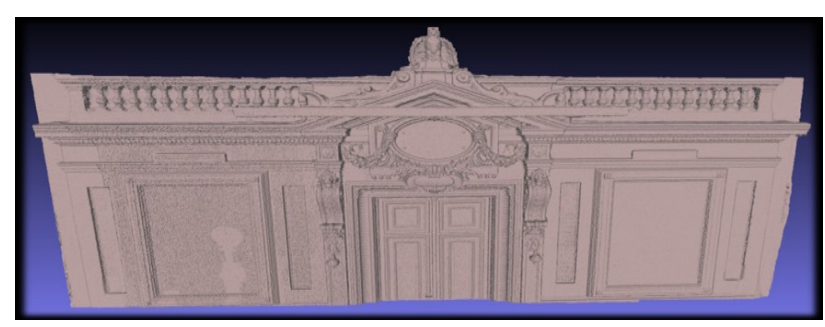

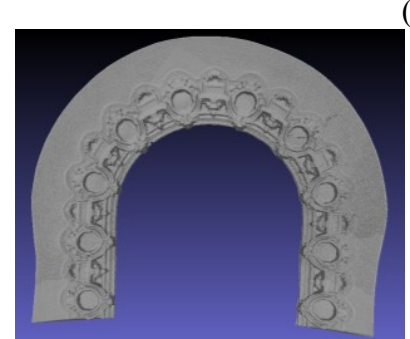

(b)

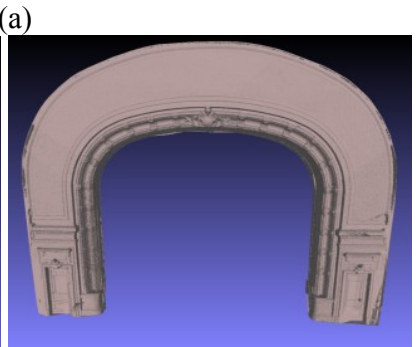

(c)
Figure 18. Mesh surface representing the (a) wall from the back of the presidium area; (b) ceiling; (c) the arch that frames the presidium area

\section{CONCLUSION}

In this paper, the accuracy of a HMLS point cloud have been evaluated, performing a comparison with a TLS point cloud in terms of two well-known distances, $\mathrm{M} 3 \mathrm{C} 2$ and the Hausdorff distance, respectively and a comparison between the extracted cross-sections. Also, the degree of noise of the HMLS point cloud was determined by finding the best fitting geometrical shapes, like sphere and plane, concluding that the standard deviation of the perpendiculars from each point and the best fitting shapes is in range of $1-1.5 \mathrm{~cm}$ and the maximum value of the perpendiculars is approximately $5 \mathrm{~cm}$.

Evaluating the accuracy of two registration methods, a semiautomatic one based on 3D conformal transformation and common targets and a completely automatic one based on ICP algorithm, we can conclude that the completely automatic registration can be used when a precision of $1-3 \mathrm{~cm}$ is required.

The M3C2 or the Hausdorff distances, can be used to identify the non-overlapping areas between two different point clouds, based on the distances distribution histogram and a defined threshold, so a complete point cloud of a scanned scene can be created with high accuracy by integration.

When comparing the cross-sections extracted from both point clouds, an average RMSE of about $1.5 \mathrm{~cm}$ has been obtained being comparable with the sigma value for the best fitting geometrical shapes.

The results are promising in using this technology for traditional outputs such as floor plans, cross sections and front elevation. Since, architects and restorers are still using the traditional outputs, this technology can be used for mapping complex scenes instead of traditional techniques, such as distometer or total station. Moreover, the HMLS technology can provide supplementary data for the TLS technology, to complete the missing parts.

\section{REFERENCES}

Bauwens, S.; Bartholomeus, H.; Calders, K.; Lejeune, P., 2016. Forest Inventory with Terrestrial LiDAR: A Comparison of Static and Hand-Held Mobile Laser Scanning. Forests, 7, 127. https://doi.org/10.3390/f7060127.

Besl, P.J.; McKay, N.D., 1992 A method for registration of 3-D shapes. IEEE Trans. Pattern Anal. Mach. Intell., 14, 239-256.

Cabo C, Del Pozo S, Rodríguez-Gonzálvez P, Ordóñez C, González-Aguilera D., 2018 Comparing Terrestrial Laser Scanning (TLS) and Wearable Laser Scanning (WLS) for Individual Tree Modeling at Plot Level. Remote Sensing., 10(4):540.

Dewez, T.J.B., Plat, E., Degas, M., Richard, T., Pannet, P., Thuon, Y., Meire, B., Watelet, J.M., Cauvin, L., Lucas, J., 2017. Handheld Mobile Laser Scanners Zeb-1 and Zeb-Revo to map an underground quarry and its above-ground surroundings. In Proceedings of the 2nd Virtual Geosciences Conference: VGC 2016, Bergen, Norway.

Girardeau-Montaut, D., 2014. CloudCompare.

http://www.danielgm.net/index.php

Gollob C, Ritter T, Nothdurft A., 2020. Forest Inventory with Long Range and High-Speed Personal Laser Scanning (PLS) and Simultaneous Localization and Mapping (SLAM) Technology. Remote Sensing, 12(9):1509.

Keawaram, B.; Dumrongchai, P.; 2017. Comparisons of Surveying with Terrestrial Laser Scanner and Total Station for Volume Determination of Overburden and Coal Excavations in 
Large Open-Pit Mine, International Journal of Geological and Environmental Engineering, Vol:11, No:12.

Maboudi, M., Bánhidi, D., Gerke, M., 2017. Evaluation of indoor mobile mapping systems. In Proceedings of the GFaI Workshop 3D-NordOst 2017 (20th Application-Oriented Workshop on Measuring, Modeling, Processing and Analysis of 3D-Data), Berlin, Germany.

Meshlab, 2013. Meshlab. http://meshlab.sourceforge.net/

Oniga, E., Savu, A., Negrilă, A., 2016 The evaluation of CloudCompare software in the process of TLS point clouds registration, Journal of Geodesy and Cadastre RevCAD, no. 21, pp. 117-124, Alba Iulia, Romania, ISSN 1583-2279.

Russhakim N. A. S., Ariff M. F. M., Majid Z., Idris K. M., Darwin N., Abbas M. A., Zainuddin K., Yusoff A. R., 2019. The suitability of terrestrial laser scanning for building survaey and mapping applications. The International Archives of the Photogrammetry, Remote Sensing and Spatial Information Sciences, Volume XLII-2/W9.

Ryding J, Williams E, Smith MJ, Eichhorn MP., 2015. Assessing Handheld Mobile Laser Scanners for Forest Surveys. Remote Sensing, 7(1):1095-1111.

Shao, J., Zhang, W., Luo, L., Cai, S., Jiang, H., 2020. SlamBased Backpack Laser Scanning for Forest Plot Mapping. ISPRS Ann. Photogramm. Remote Sens. Spat. Inf. Sci., 2, $267-$ 271.

Sirmacek, B.; Shen, Y.; Lindenbergh, R.; Zlatanova, S.; Diakite, 2016. A. Comparison of Zeb1 and Leica C10 indoor laser scanning point clouds. ISPRS Ann. Photogramm. Remote Sens. Spat. Inf. Sci., 3, 143.

ZEB Horizon-GeoSLAM. Available online: https:/geoslam.com/solutions/zeb-horizon/ 